\title{
Initial non-weight-bearing therapy is important for preventing vertebral body collapse in elderly patients with clinical vertebral fractures
}

This article was published in the following Dove Press journal:

International Journal of General Medicine

21 April 2012

Number of times this article has been viewed

\author{
Yoichi Kishikawa \\ Kishikawa Orthopaedic Clinic, \\ Saga City, Saga, Japan
}

Correspondence: Yoichi Kishikawa Kishikawa Orthopedic Surgery,

862-I Honjo, Honjo-machi,

Saga 840-0027, Japan

$\mathrm{Tel}+8 \mid 95225$ |35|

Fax +8I 95225 I367

Email yoichi@kishikawa-orthopedics.com
Purpose: The aim of the present conventional observational study was to compare the clinical outcomes of initial non-weight-bearing therapy and conventional relative rest therapy among elderly patients with clinical vertebral fractures.

Methods: In total, 196 consecutive patients with clinical vertebral fractures (mean age: 78 years) who were hospitalized for treatment between January 1999 and March 2007 were analyzed. Initial non-weight-bearing therapy consisted of complete bed rest allowing rolling on the bed without any weight-bearing to the spine for 2 weeks, followed by rehabilitation wearing a soft brace. The indications for initial non-weight-bearing therapy were vertebral fracture involving the posterior portion of the vertebral body at the thoraco-lumbar spine, mild neurological deficit, instability of the fracture site, severe pain, multiple vertebral fractures arising from trauma, malalignment at the fracture site, and mild spinal canal stenosis caused by the fracture. Patients who met the indication criteria were treated with initial non-weight-bearing therapy $(n=103)$, while the other patients were treated with conventional relative rest $(n=93)$. All the patients were uniformly treated with intramuscular elcatonin to relieve pain. The primary endpoint was progression of the vertebral fracture. The secondary endpoints included bony union and subjective back pain. The follow-up period was 12 weeks.

Results: Compared with the conventional relative rest group, the collapse rate of the anterior and posterior portions of the vertebral body was significantly smaller in the initial non-weightbearing group. The bony union rate was $100 \%$ in the initial non-weight-bearing group and $97 \%$ in the conventional relative rest group. The number of patients who experienced back pain was significantly lower in the initial non-weight-bearing group than in the conventional relative rest group.

Conclusion: These results suggest that initial non-weight-bearing therapy is important for preventing vertebral body collapse and for relieving pain among elderly patients with clinical vertebral fractures.

Keywords: clinical vertebral fracture, non-weight-bearing, brace, osteoporosis

\section{Introduction}

Osteoporosis commonly affects postmenopausal women because estrogen deficiency after menopause induces rapid bone loss. This disease increases the risk of fractures at skeletal sites such as the vertebrae, wrist, and hip. Osteoporotic vertebral and hip fractures increase morbidity and mortality among elderly men and women., ${ }^{1,2}$

Vertebral fractures are more common among Japanese elderly people than among Caucasian elderly people. ${ }^{3}$ Although most hip fractures are treated surgically if the patient's general condition permits, an appropriate therapy for vertebral fractures has 
not been established. The pain and disability caused by osteoporotic vertebral fractures have long motivated the search for effective therapy.

Recently, two procedures designed to restore vertebral body height and function have been widely adopted: percutaneous vertebroplasty, in which cement is injected into the vertebral body to support the fractured bone; and kyphoplasty, a variant of vertebroplasty in which a balloon is inserted and inflated in a collapsed vertebral body, restoring the bone's height before the cement injection. ${ }^{4}$ However, neither procedure is risk-free: reported complications include compression fractures, cement leakage, pulmonary complications, paraplegia, and death. ${ }^{5}$ Furthermore, the highest quality trials cast doubt on the benefit of the two procedures with regard to pain and disability. ${ }^{6-8}$ Therefore, these procedures should not be considered as standard treatments for osteoporotic vertebral fractures.

Strict conservative therapy should primarily be considered in patients with clinical (fresh) vertebral fractures in the absence of neurological deficits. Theoretically, nonweight-bearing to the fractured vertebral body is thought to prevent the progression of vertebral body collapse. In clinical practice, however, because conventional relative rest therapy is usually performed for clinical osteoporotic vertebral fractures among elderly patients, the effectiveness of non-weight-bearing therapy remains to be established. It was hypothesized that initial non-weight-bearing therapy would be more useful than conventional relative rest therapy for preventing vertebral body collapse and relieving pain among elderly patients with clinical vertebral fractures. The aim of the present conventional observational study was to compare the clinical outcome between initial non-weightbearing therapy and conventional relative rest therapy among elderly patients with clinical vertebral fractures.

\section{Subjects and methods Subjects}

In total, 196 consecutive elderly patients (mean age: 78 years) with clinical (painful) vertebral fractures who were hospitalized for treatment at Kishikawa Orthopedic Surgery (Saga, Japan) during a 9-year period between January 1999 and March 2007 were analyzed. The inclusion criteria were age $\geq 65$ years and a traumatic or non-traumatic clinical (fresh) vertebral fracture (T4-L5) determined based on clinical symptoms, radiographs, and magnetic resonance (MR) images of the lumbar and thoracic spine. The exclusion criteria included allergy to calcitonin and bone diseases, including bone metastases of cancers, primary hyperparathyroidism, hyperthyroidism, Cushing syndrome, multiple myeloma, and osteogenesis imperfecta. Patients who had ever taken any medications known to affect bone metabolism, including bisphosphonates, selective estrogen receptor modulators, vitamin $\mathrm{D}_{3}$, vitamin $\mathrm{K}_{2}$, or teriparatide, were also excluded because these medications might have affected the bony union of the fractures.

Initial non-weight-bearing therapy consisted of complete bed rest without any weight-bearing to the spine for 2 weeks, followed by rehabilitation wearing a soft brace. During the initial 2 weeks, even having meals and voiding were performed while lying in bed. The indications for initial nonweight-bearing therapy were vertebral fracture involving the posterior portion of the vertebral body at the thoraco-lumbar spine, mild neurological deficit, instability of the fracture site, severe pain, multiple vertebral fractures arising from trauma, malalignment (severe scoliosis or kyphosis) at the fracture site, and mild spinal canal stenosis caused by the fracture. Conventional relative rest therapy consisted of bed rest, soft brace wearing, and the minimization of weight-bearing to the spine. Patients who met the indication criteria were treated with initial non-weight-bearing therapy $(n=103)$, while the other patients were treated with conventional relative rest $(n=93)$. All the patients were uniformly treated with intramuscular elcatonin (20 units, weekly) to relieve pain, ${ }^{9,10}$ and the ordinary use of oral non-steroidal anti-inflammatory drugs (NSAIDs) was avoided to monitor pain. The primary endpoint was the progression of the vertebral fracture. The secondary endpoints included bony union and subjective back pain. The follow-up period was 12 weeks. The present study was approved by the Ethics Committee of Kishikawa Orthopedic Surgery.

\section{Assessment of prevalent vertebral fractures}

Plain lateral X-ray films of the thoracic and lumbar spine were obtained at baseline to detect evidence of morphometric vertebral fractures. According to the Japanese criteria, a vertebral fracture was defined according to the vertebral height on lateral X-ray films. ${ }^{11,12}$ Briefly, the vertebral height was measured at the anterior, central, and posterior portions of the vertebral body, and the presence of a vertebral fracture was confirmed when (1) a reduction in the vertebral height of more than $20 \%$ (anterior, central, and posterior portions) compared with the height of the adjacent vertebrae was observed, (2) the central height/anterior height or central height/posterior height was less than 0.8 , or (3) the anterior 
height/posterior height was less than 0.75 . The assessment for vertebral fractures was performed at the T4-L5 level.

\section{Evaluation of clinical vertebral fractures}

Radiographs and MR images of the lumbar and thoracic spine were obtained at baseline to detect evidence of vertebral fractures at the T4-L5 level. Plain anterior-posterior and lateral X-ray films of the lumbar and thoracic spine were taken while the patient was in both a standing and a supine position. Alterations in the vertebral fractures were assessed every 2 weeks for 12 weeks.

The collapse rate of the anterior and posterior portions was evaluated using the following formula (Figure 1): $\Delta \mathrm{C}=\mathrm{C} / \mathrm{E}$ and $\Delta \mathrm{D}=\mathrm{D} / \mathrm{E}$, respectively, where $\mathrm{A}$ is the height of the posterior portion of the upper intact vertebral body, $\mathrm{B}$ is the height of the posterior portion of the lower intact vertebral body, $\mathrm{C}$ is the height of the anterior portion of the fractured vertebral body, $\mathrm{D}$ is the height of the posterior portion of the fractured vertebral body, and $\mathrm{E}$ is $(\mathrm{A}+\mathrm{B}) / 2$.

Local alignment was evaluated according to the angle with the posterior portion's lines (A and B in the standing position) for the upper and lower vertebral body (Figure 1). An angle $\geq 5$ degrees lordotic was judged as lordosis (score: -1 ), an angle $\geq 5$ degrees kyphotic was judged as kyphosis (score: +1 ), and angles between -5 to 5 degrees were judged as being straight (score: 0 ). The sum of the scores was used to evaluate local alignment.

The severity of the vertebral fracture was evaluated according to the fracture type: burst fracture (score: +1$)$,

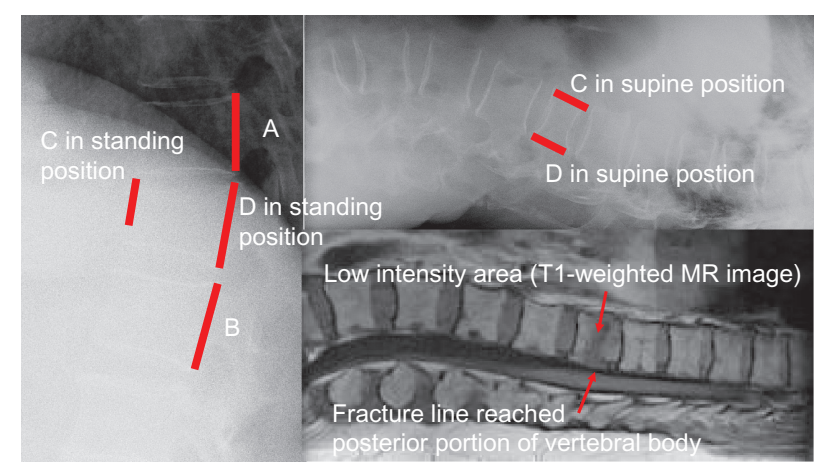

Figure I Evaluation of radiographs and MRI.

Notes: On radiographs, fractures were evaluated using the following parameters: $A=$ height of posterior portion of the upper intact vertebral body, $B=$ height of posterior portion of the lower intact vertebral body, $C=$ height of anterior portion of the fractured vertebral body, $D=$ height of posterior portion of the fractured vertebral body, $\mathrm{E}=(\mathrm{A}+\mathrm{B}) / 2, \Delta \mathrm{C}=\mathrm{C} / \mathrm{E}, \Delta \mathrm{D}=\mathrm{D} / \mathrm{E}$. On TI-weighted MR images, fractures were evaluated by the fracture line reached posterior portion of the vertebral body, posterior portion of the vertebral body collapsed, and low intensity area $\geq 50 \%$ of the vertebral body.

Abbreviation: MRI, magnetic resonance imaging. and compression fracture (score: 0 ). The sum of the scores was used to evaluate the severity of the vertebral fracture.

The severity of the vertebral fracture was also evaluated using MR images according to the fracture line, collapse of the posterior portion of the vertebral body, and T1-weighted low-intensity area (Figure 1). Fractures were judged as severe when the fracture line reached the posterior portion of the vertebral body (score: +1$)$, the posterior portion of the vertebral body had collapsed (score: +1$)$, and the T1-weighted low-intensity area accounted for $\geq 50 \%$ of the vertebral body (score: +1 ). The sum of the scores was used to evaluate the severity of the vertebral fracture.

Bony union on radiographs was confirmed when the progression of the vertebral body collapse arrested, the callus became homogeneous, and the vertebral body did not show any changes in shape between the standing and supine positions.

\section{Statistical analysis}

All the data were expressed as the mean \pm standard deviation (SD) in the tables. Data comparisons between the two groups were performed using an unpaired $t$-test and a Fisher exact test. A two-way analysis of variance (ANOVA) with repeated measurements was used to compare longitudinal changes in parameters between the two groups. All the statistical analyses were performed using the Stat View-J5.0 program (SAS Institute, Cary, NC) on a Windows computer. A significance level of $P<0.05$ was used for all the comparisons.

\section{Results \\ Characteristics of the study subjects at the start of therapy}

Table 1 shows the characteristics of the study subjects at the start of therapy. Of the 196 patients, 103 (12 men and 91 women) underwent initial non-weight-bearing therapy and 93 (20 men and 73 women) underwent conventional relative rest therapy. The patient age was significantly lower in the initial non-weight-bearing group than in the conventional relative rest group (77.7 years versus 78.6 years). The number of prevalent vertebral fractures did not differ significantly between the two groups. The time from the onset of back pain (fracture) to hospitalization was significantly shorter in the initial non-weight-bearing group than in the conventional relative rest group (9.5 days versus 19.5 days). The total number of incident vertebral fractures was 110 in the initial non-weight-bearing group and 101 in the conventional relative rest group. Regarding local alignment and the severity of the vertebral fracture, 
Table I Characteristics of study subjects at the start of therapy

\begin{tabular}{|c|c|c|c|}
\hline & Initial non-weight-bearing & Conventional relative rest & $P$ value \\
\hline Number of subjects & 103 & 93 & \\
\hline \multicolumn{4}{|l|}{ Gender } \\
\hline Male & 12 & 20 & NS \\
\hline Female & 91 & 73 & NS \\
\hline Age (years) & $77.7 \pm 6.2$ & $78.6 \pm 6.4$ & $<0.05$ \\
\hline Number of subjects with prevalent vertebral fractures & 16 & 15 & NS \\
\hline Time from onset of pain to hospitalization (days) & $9.5 \pm 19.8$ & $19.5 \pm 25.0$ & $<0.05$ \\
\hline Number of incident fractures & 110 & 101 & \\
\hline Local alignment score & $0.64 \pm 0.67$ & $0.36 \pm 0.70$ & $<0.05$ \\
\hline Severity of fractures by type of fractures & $0.28 \pm 0.45$ & $0.17 \pm 0.38$ & $<0.05$ \\
\hline Severity of fractures by MR images & $2.32 \pm 0.84$ & $1.65 \pm 1.21$ & $<0.05$ \\
\hline
\end{tabular}

Notes: Data are expressed as the mean \pm SD. An unpaired $t$-test and a Fisher exact test were used to compare data between the two groups.

Abbreviation: NS, not significant.

alignment was more significantly altered after vertebral fracture in the initial non-weight-bearing group than in the conventional relative rest group, and the severity of the vertebral fracture evaluated according to the fracture type and MR images was greater in the non-weight-bearing group than in the conventional relative rest group.

The causes of the vertebral fractures were falling (49.1\%), tumbling (7.3\%), lifting (14.5\%), other minor trauma $(14.5 \%)$, and no trauma $(14.5 \%)$ in the initial nonweight-bearing group, and falling (38.6\%), tumbling (1.0\%), other minor trauma (34.7\%), traffic accident (1.0\%), and no trauma $(24.8 \%)$ in the conventional relative rest group.

Figure 2 shows the distribution of the clinical vertebral fractures in the initial non-weight-bearing and conventional relative rest groups. Vertebral fractures at the thoraco-lumbar spine (T12 and L1) with kyphosis were more likely to be treated with initial non-weight-bearing therapy, whereas vertebral fractures at the thoraco-lumbar (T12 and L1) and upper to middle thoracic spine with kyphosis and those at the lower lumbar spine with lordosis were more likely to be treated with conventional relative rest therapy.

\section{Changes in height of the anterior and posterior potions of the vertebral body and bony union rate}

The changes in $\Delta \mathrm{C}$ and $\Delta \mathrm{D}$ differed significantly between the two groups (both $P<0.05$ according to a two-way ANOVA with repeated measurements) (Figure 3 ). $\Delta \mathrm{C}$ and $\Delta \mathrm{D}$ were significantly greater at 2,6 , and 12 weeks in the initial nonweight-bearing group than in the conventional relative rest group ( $P<0.01$ for $\Delta \mathrm{C}$ and $P<0.05$ for $\Delta \mathrm{D}$ at 2 weeks and $P<0.05$ for $\Delta \mathrm{C}$ and $\Delta \mathrm{D}$ at 6 and 12 weeks according to an unpaired $t$-test). These results suggested that the collapse rate (the height loss) of the anterior and posterior portions of the vertebral body was significantly smaller in the initial non-weight-bearing group than in the conventional relative rest group.

After 12 weeks of therapy, the bony union rate was $100 \%$ in the initial non-weight-bearing group and $97 \%$ in
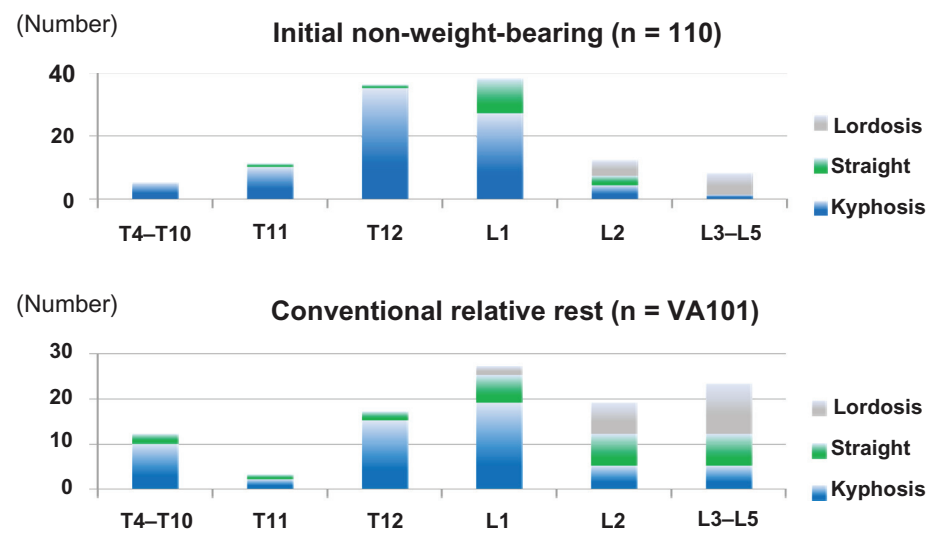

Figure 2 Distribution of vertebral fractures according to type of therapy.

Notes: Vertebral fractures at the thoraco-lumbar spine (TI2 and LI) with kyphosis were more likely to be treated with initial non-weight-bearing therapy, whereas vertebral fractures at the thoraco-lumbar (TI2 and LI) and upper to middle thoracic spine with kyphosis and those at the lower lumbar spine with lordosis were more likely to be treated with conventional relative rest therapy. 

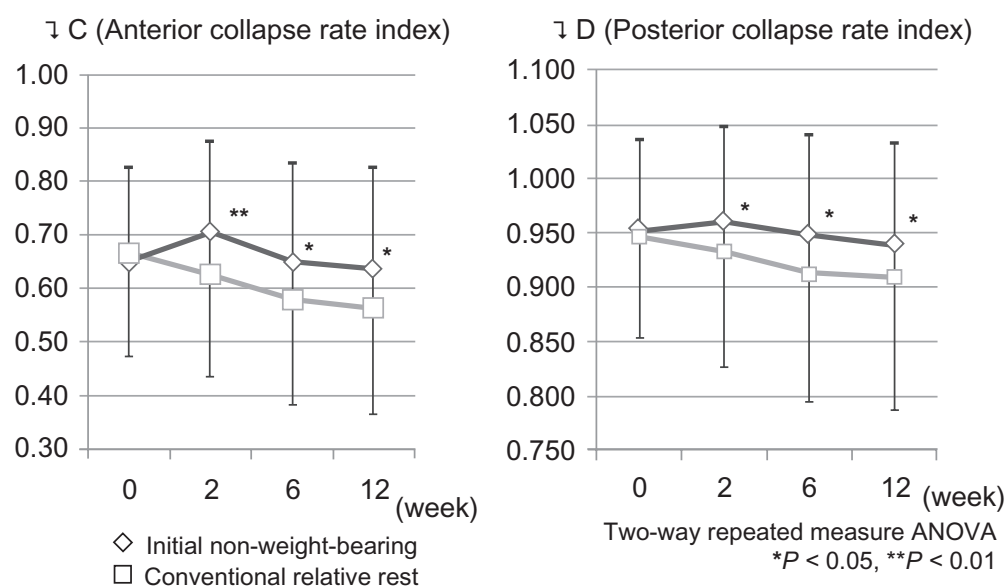

Figure 3 Changes in collapse rate index of anterior and posterior portion of vertebral body.

Notes: The changes in $\Delta \mathrm{C}$ and $\triangle \mathrm{D}$ differed significantly between the two groups (both $P<0.05$ according to a two-way ANOVA with repeated measurements). $\triangle \mathrm{C}$ and $\triangle \mathrm{D}$ were significantly greater at 2,6 , and 12 weeks in the initial non-weight-bearing group than in the conventional relative rest group. $* P<0.05$, $* * P<0.0 \mathrm{I}$ versus conventional relative rest group by the unpaired $t$-test.

Abbreviation: ANOVA, analysis of variance.

the conventional relative rest group. In particular, a subgroup analysis of patients among whom treatment was initiated within 2 weeks of onset revealed that the bony union rates at 6 and 12 weeks were significantly greater in the initial non-weight-bearing group than in the conventional relative rest group (6 weeks: $80.0 \%$ versus $40.7 \%$, 12 weeks: $85.7 \%$ versus $51.4 \%$; both $P<0.01$ according to a Fisher exact test) (Figure 4).

A subgroup analysis among patients who suffered from thoraco-lumbar vertebral fractures showed that the fracture was repositioned in approximately $10 \%$ of the patients in the initial non-weight-bearing group (from $\Delta \mathrm{C}<0.5$ to $\Delta \mathrm{C}>0.5$ ) and that the collapse progressed in approximately $30 \%$ of the patients in the conventional relative rest group (from $\Delta \mathrm{C}>0.5$ to $\Delta \mathrm{C}<0.5$ ).

\section{Changes in subjective back pain and hospitalization period}

The percentages of patients who sustained no back pain, mild back pain, and moderate back pain were $37 \%, 62 \%$, and $1 \%$, respectively, in the initial non-weight-bearing group and $12 \%, 75 \%$, and $13 \%$, respectively, in the conventional

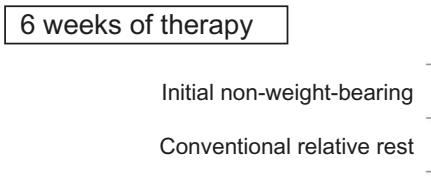

Bony union rate (\%)
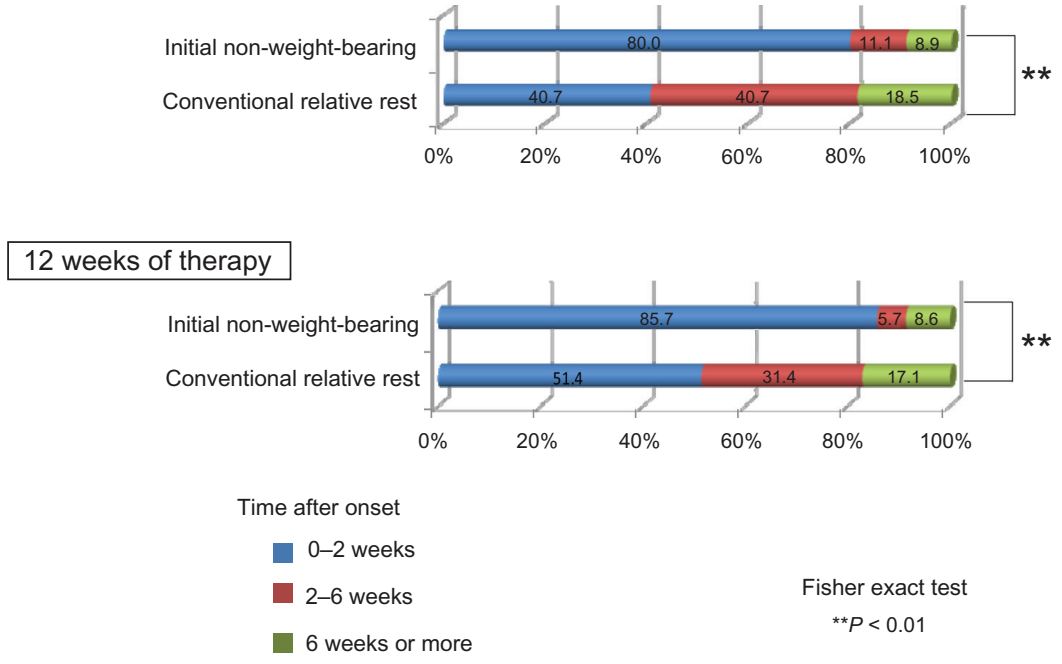

Figure 4 Bony union rate according to time after onset.

Notes: A subgroup analysis in patients who were initiated therapy by 2 weeks after onset, bony union rate at 6 and 12 weeks was significantly greater in the initial non-weightbearing group than in the conventional relative rest group. $* P<0.05$, $* * P<0.0$ I versus conventional relative rest group by the Fisher's exact test. 
relative rest group. The percentage of patients who sustained no back pain was significantly greater in the initial nonweight-bearing group than in the conventional relative rest group ( $P<0.001$ according to a Fisher exact test), and the percentage of patients who sustained moderate back pain was significantly smaller in the initial non-weight-bearing group than in the conventional relative rest group $(P<0.01$ according to a Fisher exact test).

The hospitalization period was significantly longer in the initial non-weight-bearing group than in the conventional relative rest group (49.2 days versus 37.9 days; $P<0.05$ according to an unpaired $t$-test).

\section{Complications}

The main complications of initial non-weight-bearing therapy were constipation (45\%), dizziness (25\%), nausea or vomiting (6\%), cystitis ( $2 \%)$, and intercostal neuralgia $(2 \%)$. Nausea, vomiting, and dizziness were transiently observed at the initiation of rehabilitation and disappeared thereafter. No serious adverse events were observed.

\section{Representative case}

Figure 5 shows the representative case of an 80-year-old woman who suffered a T12 burst fracture (MR image).
Initial non-weight-bearing therapy was performed. After 12 weeks of therapy, bony union was confirmed using radiographs obtained while the patient was in both a standing and a supine position.

\section{Discussion}

The aim of the present conventional observational study was to compare the clinical outcomes between initial non-weightbearing therapy and conventional relative rest therapy among elderly patients with clinical vertebral fractures. Compared with the conventional relative rest group, the collapse rate (the height loss) of the anterior and posterior portions of the vertebral body after 12 weeks of therapy was significantly smaller in the initial non-weight-bearing group. These results suggest that initial non-weight-bearing therapy is important for preventing vertebral body collapse in elderly patients with clinical vertebral fractures.

Delmas et $\mathrm{al}^{13}$ analyzed data from the randomized, doubleblinded 3-year Multiple Outcomes of Raloxifene Evaluation (MORE) trial. Among women without prevalent vertebral fractures and with mild, moderate, and severe prevalent vertebral fractures, $4.3 \%, 10.5 \%, 23.6 \%$, and $38.1 \%$, respectively, had new vertebral fractures, whereas $5.5 \%, 7.2 \%, 7.7 \%$, and $13.8 \%$, respectively, experienced new nonvertebral fractures.

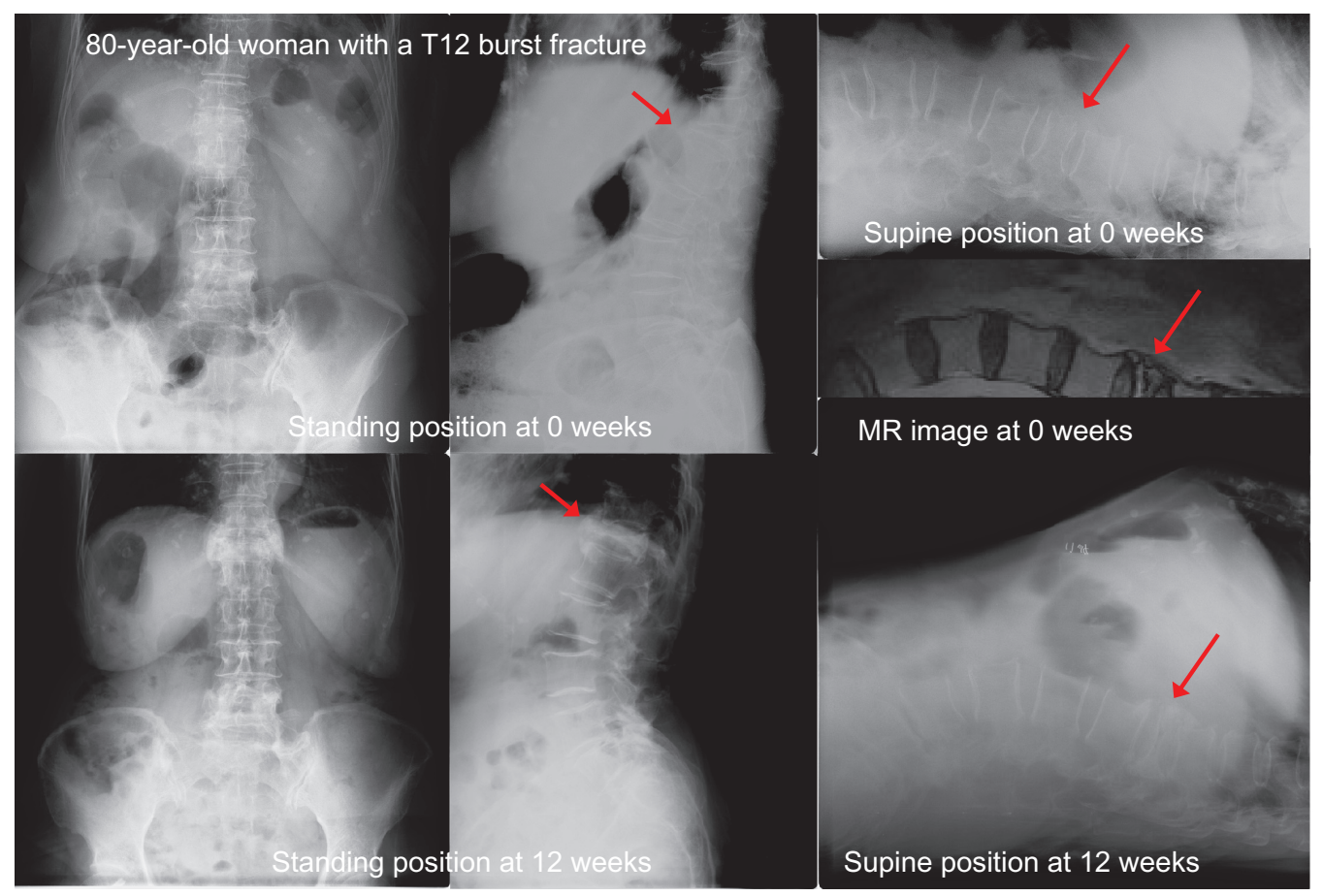

Figure 5 Bony union confirmed by standing and supine position radiographs.

Notes: Initial non-weight-bearing therapy was performed. Bony union on radiographs was confirmed when progression of vertebral body collapse arrested, callus became homogeneous, and vertebral body form did not show any changes between standing and supine positions. After 12 weeks of therapy, bony union was confirmed by radiographs in the standing and supine position. 
Thus, vertebral fracture severity appears to be the best independent predictor for the risk of new vertebral and nonvertebral fractures. Miyakoshi et al ${ }^{14,15}$ reported that the quality of life (QOL) in patients with osteoporosis was impaired by postural deformities, especially by whole kyphosis, and that increases in lumbar kyphosis and the number of vertebral fractures represented very important risk factors for gastroesophageal reflux disease (GERD) in patients with osteoporosis. These reports suggest that the prevention of severe vertebral fractures is very important for preventing subsequent fractures, avoiding deterioration in the QOL, and reducing the risk of GERD in patients with osteoporosis.

Fractures involving the posterior portion of the thoracolumbar vertebral body may potentially cause cord compression, possibly resulting in paralysis in severe cases. The indications for initial non-weight-bearing therapy included vertebral fractures involving the posterior portion of the vertebral body at the thoraco-lumbar spine, mild neurological deficit, instability of the fracture site, and mild spinal canal stenosis caused by the fracture. Despite the severer vertebral fractures, compared with the conventional relative rest group, the risk of vertebral body collapse was lower in the initial non-weight-bearing group.

Other advantages of initial non-weight-bearing therapy in patients with clinical vertebral fractures included an excellent bony union rate when the therapy was initiated within 2 weeks after the onset of pain, possible repositioning of the fracture, and a pain relief effect. The disadvantages were a long hospitalization period and complications such as constipation, dizziness, nausea or vomiting, cystitis, and intercostal neuralgia, all of which were transient. The advantages appeared to outweigh the disadvantages.

We took radiographs every 2 weeks to observe the bony union process. As shown in Figure 6, callus formation was found around the pedicle in the posterior part of the vertebral body as early as 2 weeks after the initiation of initial nonweight-bearing therapy. Then, callus formation was extended to the anterior part of the vertebral body at 4 weeks. The callus became homogeneous according to the progression of the bony union at 8 weeks, and a bridging callus also formed to stabilize the fractured vertebra with endplate injury. Therefore, non-weight-bearing therapy was required to avoid the impairment of bone union. None of the cases experienced the failure of bony union in terms of pseudoarthrosis after the initial non-weight-bearing therapy.

Intramuscular treatment with elcatonin is commonly used in Japan. Several studies have shown that intramuscular elcatonin is useful for relieving pain and improving the QOL of postmenopausal Japanese women with or without osteoporosis., ${ }^{9,16}$ The evidence-based clinical practice guidelines of the American Academy of Orthopedic Surgeons (AAOS) suggest that calcitonin therapy should be recommended for the treatment of patients who present with a clinical osteoporotic spinal compression fracture (strength of recommendation is moderate).${ }^{10}$ A preclinical study showed that elcatonin did not interfere with bone healing in a femoral fracture model in cynomolgus monkeys. ${ }^{17}$ Therefore, elcatonin may primarily be used for pain relief in patients with osteoporotic clinical vertebral fracture.

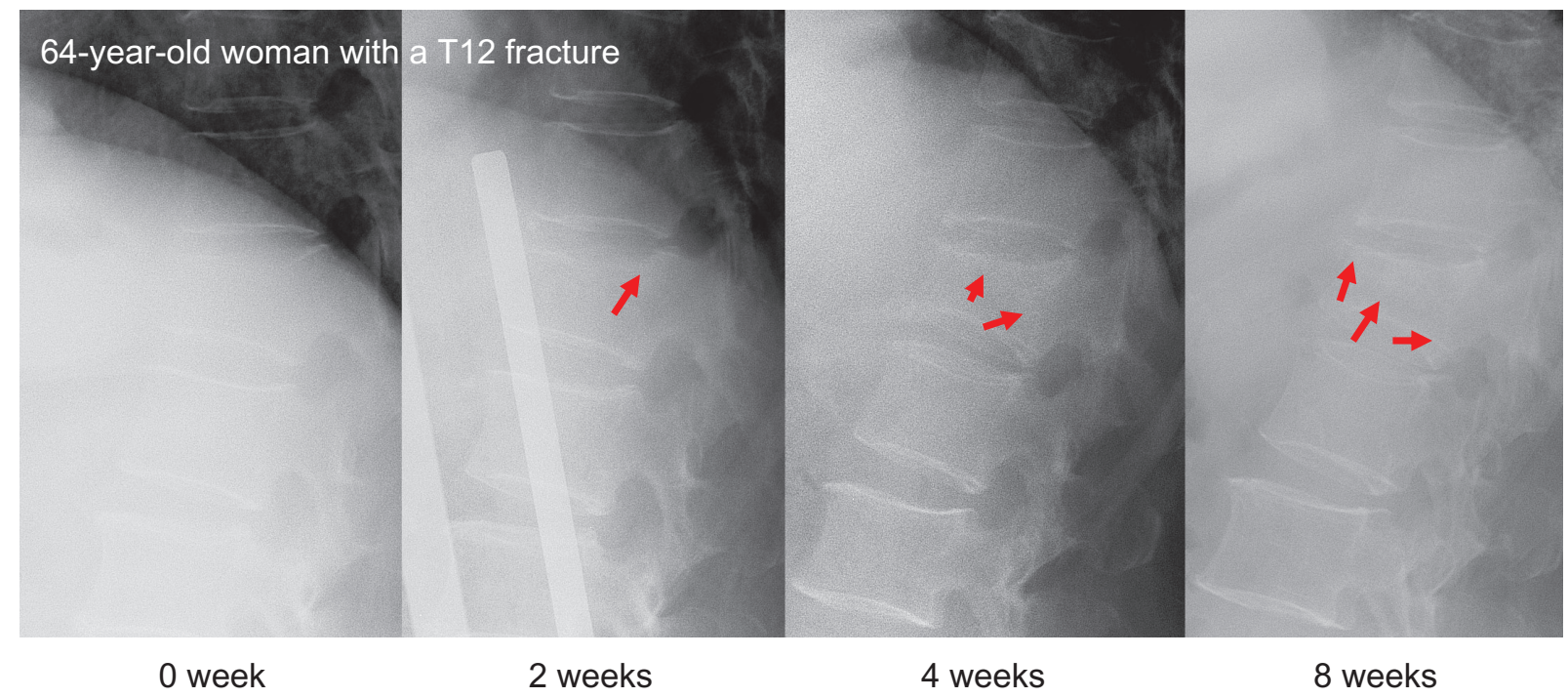

Figure 6 Process of bony union: representative case.

Notes: Callus formation was found around the pedicle in the posterior part of the vertebral body as early as 2 weeks after the initiation of initial non-weight-bearing therapy. Then, callus formation was extended to the anterior part of the vertebral body at 4 weeks. The callus became homogeneous according to the progression of bony union at 8 weeks and a bridging callus was also formed to stabilize the fractured vertebra with endplate injury. 
The present study had some notable limitations. First, the present study was a conventional observational study, and the type of vertebral fractures was not uniform between the two groups. Namely, the severity of the vertebral fractures at the initiation of the therapy was greater in the initial weightbearing group than in the conventional relative rest group. Thus, the results might be biased. Second, age at baseline was significantly lower in the initial non-weight-bearing group (mean: 77.7 years) than in the conventional relative rest group (mean: 78.6 years). However, because the difference in age at baseline was only 0.9 years, it was surmised that this difference might not have strongly influenced the results of the present study. Third, pain was not assessed quantitatively using a visual analogue scale. Fourth, function (Roland-Morris Disability Questionnaire) $)^{18-20}$ and QOL (SF-36) were not examined, although these parameters are important in elderly patients. Fifth, the follow-up period was only 12 weeks and longer periods of observation are needed to determine the effectiveness of initial non-weight-bearing therapy. RCTs are needed to confirm the long-term effectiveness of initial non-weight-bearing therapy in patients with clinical vertebral fractures. This paper may stimulate further research to clarify the effectiveness of conservative treatment for clinical vertebral fractures.

In conclusion, the results of the present study suggest that initial non-weight-bearing therapy is important for preventing vertebral body collapse and relieving pain among elderly patients with clinical vertebral fractures.

\section{Disclosure}

The author reports no funding sources or conflict of interest in this work.

\section{References}

1. Epstein S. Postmenopausal osteoporosis: fracture consequences and treatment efficacy vary by skeletal site. Aging (Milano). 2000;12(5): 330-341.

2. Bliuc D, Nguyen ND, Milch VE, Nguyen TV, Eisman JA, Center JR. Mortality risk associated with low-trauma osteoporotic fracture and subsequent fracture in men and women. JAMA. 2009;301(5):513-521.
3. Fujiwara S, Kasagi F, Masunari N, Naito K, Suzuki G, Fukunaga M. Fracture prediction from bone mineral density in Japanese men and women. J Bone Miner Res. 2003;18(8):1547-1553.

4. Elshaug AG, Garber AM. How CER could pay for itself-insights from vertebral fracture treatments. N Engl J Med. 2011;364(15):1390-1393.

5. Manufacturer and User Facility Device Experience Database (MAUDE). Silver Spring, MD: Food and Drug Administration. http://www.fda. gov/MedicalDevices/DeviceRegulationandGuidance/Postmarket Requirements/ReportingAdverseEvents/ucm127891.htm.

6. Weinstein JN. Balancing science and informed choice in decisions about vertebroplasty. $N$ Engl J Med. 2009;361(6):619-621.

7. Kallmes DF, Comstock BA, Heagerty PJ, et al. A randomized trial of vertebroplasty for osteoporotic spinal fractures. $N$ Engl J Med. 2009; 361(6):569-579.

8. Staples MP, Kallmes DF, Comstock BA, et al. Effectiveness of vertebroplasty using individual patient data from two randomised placebo controlled trials: meta-analysis. BMJ. 2011;343:d3952.

9. Ikegami S, Kamimura M, Uchiyama S, et al. Anti-nociceptive effects of elcatonin injection for postmenopausal women with back pain: a randomized controlled trial. Open Orthopa J. 2010;4:132-136.

10. American Academy of Orthopaedic Surgeons. Clinical Practice Guidelines. Available from: http://www.aaos.org/Research/guidelines/ guide.asp.

11. Orimo H, Sugioka Y, Fukunaga M, et al. Diagnostic criteria of primary osteoporosis. J Bone Miner Metab. 1998;16:139-150.

12. Orimo H, Hayashi Y, Fukunaga M, et al. Diagnostic criteria for primary osteoporosis: year 2000 revision. J Bone Miner Metab. 2001; 19(6):331-337.

13. Delmas PD, Genant HK, Crans GG, et al. Severity of prevalent vertebral fractures and the risk of subsequent vertebral and nonvertebral fractures: results from the MORE trial. Bone. 2003;33(4):522-532.

14. Miyakoshi N, Itoi E, Kobayashi M, et al. Impact of postural deformities and spinal mobility on quality of life in postmenopausal osteoporosis. Osteoporos Int. 2003;14(12):1007-1012.

15. Miyakoshi N, Kasukawa Y, Sasaki H, Kamo K, Shimada Y. Impact of spinal kyphosis on gastroesophageal reflux disease symptoms in patients with osteoporosis. Osteoporos Int. 2009;20(7):1193-1198.

16. Yoh K, Tanaka K, Ishikawa A, et al. Health-related quality of life (HRQOL) in Japanese osteoporotic patients and its improvement by elcatonin treatment. J Bone Miner Metab. 2005;23(2):167-173.

17. Manabe T, Mori S, Mashiba T, et al. Eel calcitonin (elcatonin) suppressed callus remodeling but did not interfere with fracture healing in the femoral fracture model of cynomolgus monkeys. $J$ Bone Miner Metab. 2009;27(3):295-302.

18. Roland M, Fairbank J. The Roland-Morris Disability Questionnaire and the Oswestry Disability Questionnaire. Spine (Phila Pa 1976). 2000;25(24):3115-3124.

19. Roland MO, Morris RW. A study of the natural history of back pain. Part 1:Development of a reliable and sensitive measure of disability in low back pain. Spine. 1983;8(2):141-144.

20. Suzukamo Y, Fukuhara S, Kikuchi S, et al. Validation of the Japanese version of the Roland-Morris Disability Questionnaire. J Orthop Sci. 2003;8(4):543-548.
International Journal of General Medicine

\section{Publish your work in this journal}

The International Journal of General Medicine is an international, peer-reviewed open-access journal that focuses on general and internal medicine, pathogenesis, epidemiology, diagnosis, monitoring and treatment protocols. The journal is characterized by the rapid reporting of reviews, original research and clinical studies across all disease areas.

\section{Dovepress}

A key focus is the elucidation of disease processes and management protocols resulting in improved outcomes for the patient.The manuscript management system is completely online and includes a very quick and fair peer-review system. Visit http://www.dovepress.com/ testimonials.php to read real quotes from published authors. 